Tohoku J. Exp. Med., 2007, 212, 35-41

\title{
Correlation between Clinicopathology and Expression of Heat Shock Protein 72 and Glycoprotein 96 in Human Gastric Adenocarcinoma
}

\author{
Xiao-Ping Wang, ${ }^{1}$ Qiao-Xia Wang ${ }^{1}$ and Xiao-Ping Ying ${ }^{1}$ \\ ${ }^{1}$ Department of Pathology, Shaanxi University of Chinese Medicine, Shaanxi, China
}

\begin{abstract}
WAng, X.-P., WAnG, Q.-X. and Ying, X.-P. Correlation between Clinicopathology and Expression of Heat Shock Protein 72 and Glycoprotein 96 in Human Gastric Adenocarcinoma. Tohoku J. Exp. Med., 2007, 212 (1), 35-41 — Heat shock protein 72 (HSP72) and glycoprotein 96 (gp96) are highly expressed in cancer tissues. Recent studies indicate the possible roles of HSP72 and gp96 in the development and progression of gastric carcinomas but detailed information is still ambiguous. In this study, we investigated the correlation between clinicopathology and expression of HSP72 and gp96 in human gastric carcinoma. The expression of HSP72 and gp96 was studied in 60 human gastric carcinomas with or without metastasis as well as in mucous membrane adjacent to cancers by way of immunohistochemistry. HSP72 immunoreactivities were detected in 54 of 60 primary tumors $(90.0 \%)$ and in 22 of 60 mucous membranes adjacent to cancers $(36.7 \%)$. Likewise, gp96 immunoreactivities were detected in 49 cases of gastric carcinoma (81.7\%) and in 15 samples of mucous membrane adjacent to cancer (25.0\%). Both HSP72 and gp96 were stained in cytoplasm. HSP72 and gp96 expression in colonic carcinomas with metastasis was significantly higher than those with non-metastasis $(p<0.05)$. The results indicate that there exists a significant correlation between the expression of HSP72 and gp96 and the progression of gastric carcinomas. The high-level expression of HSP72 and gp96 may be used as diagnostic or prognostic markers for gastric carcinoma. — heat shock protein 72 (HSP72); glycoprotein 96 (gp96); gastric adenocarcinoma; clinicopathology; prognosis

(C) 2007 Tohoku University Medical Press
\end{abstract}

The heat shock protein (HSP) family is a highly conserved group of cellular proteins and is up-regulated under stress conditions, such as heat, hypoxia, serum deprivation, neoplasia and virus infection (Schlesinger 1990; Morimoto 1993; Argon and Simen 1999). It functions as molecular chaperone and biochemical regulator to mediate cell growth, apoptosis, protein homeostasis and cellular targets of peptides (Morimoto 1993).
Studies have shown that HSP expression have a close relationship with carcinoma prognosis (Garcia-Bermejo et al. 1997; Lebret et al. 2003). They may combine with oncogene products to form complexes and transport them into intracellular special sites and promote cancer cell proliferation and heterogeneous differentiation (Villaseca et al. 1997; Dorsey and Tchounwou 2003). Recent studies have shown that HSP72

Received December 19, 2006; revision accepted for publication March 14, 2007.

Correspondence: Wang Xiaoping, Ph.D., Department of Pathology, Shaanxi University of Chinese Medicine, Xianyang Century Avenue, Xianyang 712046, Shaanxi, China.

e-mail: wxpphd@yahoo.com.cn 
and gp96 are highly expressed in cancer tissues and have been used as prognostic markers in some tumors (Bausero et al. 2004; Gabai et al. 2005; Wang et al. 2005b).

An earlier study indicates the possible roles of HSP72 and gp96 in the development and progression of gastric carcinomas but detailed information is still ambiguous (Maehara et al. 2000). Gastric carcinoma is one of the most malignant cancers and there may be a correlation between the progression of gastric carcinoma and overexpression of HSPs. In this study, we use immunochemical staining methods to detect the expression of HSP72 and gp96 in gastric caner, mucous membrane adjacent to cancer and gastric carcinoma tissue with or without metastasis in order to explore the relationship between these molecules and their significances. The results indicate that there exists a significant correlation between the expression of HSP72 and gp96 and the progression in gastric carcinoma.

\section{Materials And Methods}

\section{Immunochemistry reagents}

Mouse anti-human HSP72 monoclonal antibody was obtained from StressGen Biotechnologies (Victoria, British Columbia, Canada) and mouse anti-human gp96 monoclonal antibody was purchased from Santa Cruz Biotechnology, Inc. (Santa Cruz, CA, USA). EnVisionTM kits were purchased from Dako Co. (Carpinteria, CA, USA).

\section{Tissue samples}

This investigation was approved by the Ethics Committee on Human Study at Shaanxi University of Chinese Medicine (2004-4B). Paraffin specimens of primary gastric carcinoma from 60 patients undergoing gastric resection were collected from the affiliated Hospital, Shaanxi University of Chinese Medicine, Xianyang, China from 2000 to 2003. The patients consisted of 32 males and 28 females, with a mean age of 46.5 years, ranging from 28 to 74 years. Routine pathological diagnosis showed that all cases were adenocarcinoma. Twenty-seven cases were well-differentiated type (grade I) and 20 cases were moderate-differentiated type (grade II), while 13 cases were poorly differentiated (grade III). Among the cases, 45 cases had regional lymph node metastases, and 34 cases had remote metastases. The specimens were fixed in $10 \%$ buffered formalin and embedded in paraffin. Serial sections, 5 - $\mu$ m-thick, were cut and placed on silane-coated glass slides.

\section{Staining methods}

All sections were deparaffinized and rehydrated with graded alcohols. Endogenous peroxidase was then blocked with $3 \mathrm{ml} / 1 \mathrm{H}_{2} \mathrm{O}_{2}$ diluted in methanol for $30 \mathrm{~min}$ at room temperature. Antigen retrieval was performed by treating the slides in citrate buffer in a microwave for $10 \mathrm{~min}$. The slides were incubated in a moist chamber with HSP72 mouse monoclonal antibody $(1: 100)$ and gp96 mouse monoclonal antibody $(1: 100)$ at $4^{\circ} \mathrm{C}$ overnight. After a complete wash in phosphate buffered saline (PBS), the slides were incubated with horseradish peroxidase labelled goat anti-mouse antibody $(1: 100)$ for $45 \mathrm{~min}$ at $37^{\circ} \mathrm{C}$. After a complete wash in PBS, the slides were developed in $0.5 \mathrm{~g} / 1$ freshly prepared $3,3^{\prime}$ diaminobenzedine solution (DAB, Sigma Co., St. Louis, Mo, USA) for $8 \mathrm{~min}$, and then counterstained with hematoxylin, dehydrated, air dried, and mounted. Normal mouse $\operatorname{IgG}$ was used to substitute for the primary antibody as a negative control. No specific immunoreactivity was detected in these tissue sections. Two of the authors initially determined the fields simultaneously using a double-headed light microscope. The evaluation of HSP72 and gp96 positive cells was performed on high-power fields $(\times 400)$ using a standard light microscope. Only distinctive intranuclear or intra-cytoplasm immunoreactivity was considered positive. In each case, more than 500 cells were counted and the percentage of immunoreactivity was independently determined. When interobserver difference was greater than $5 \%$, the immunostained slides were re-examined simultaneously using a double-headed light microscope and the percentage of positive cells was determined. When interobserver difference was less than 5\%, the mean value was obtained as the positive rate. When more than $10 \%$ positive cells were detected, the case was considered positive.

\section{Statistical analysis}

The differences in HSP72 and gp96 expression between gastric carcinomas and mucous membrane adjacent to cancer were analyzed statistically using u test. The relationship between expression of HSP72 and gp96 in gastric carcinoma tissue with or without metastasis was analyzed statistically using $\chi^{2}$ test. $P<0.05$ was considered statistically significant. 


\section{RESUlts}

Expression of HSP72 and gp96 in gastric carcinomas and mucous membrane adjacent to cancer

The results of immunohistochemistry of HSP72 and gp96 were summarized in Table 1. HSP72 immunoreactivities were detected in 54 of 60 primary tumors $(90.0 \%)$ and in 22 of 60 mucous membranes adjacent to cancers $(36.7 \%)$. Likewise, gp96 was detected in 49 cases of gastric carcinoma $(81.7 \%)$ and in 15 samples of mucous membrane adjacent to cancer $(25.0 \%)$. Both HSP72 and gp96 stained in cytoplasm (Fig. 1).
HSP72 and gp96 positive rates in gastric carcinoma groups were significantly higher than those in mucous membrane adjacent to cancer $(p<0.01)$.

Relationship between clinicopathology and expression of HSP72 and gp96 in gastric carcinomas

HSP72 and gp96 were expressed higher in low differentiation of gastric carcinomas than those in tissues adjacent to cancers (Table 1) $(p<$ $0.01)$. HSP72 and gp96 positive rates in lymph node metastasis and remote metastasis groups were $100 \%$. There were significant differences in HSP72 and gp96 expression between metastasis
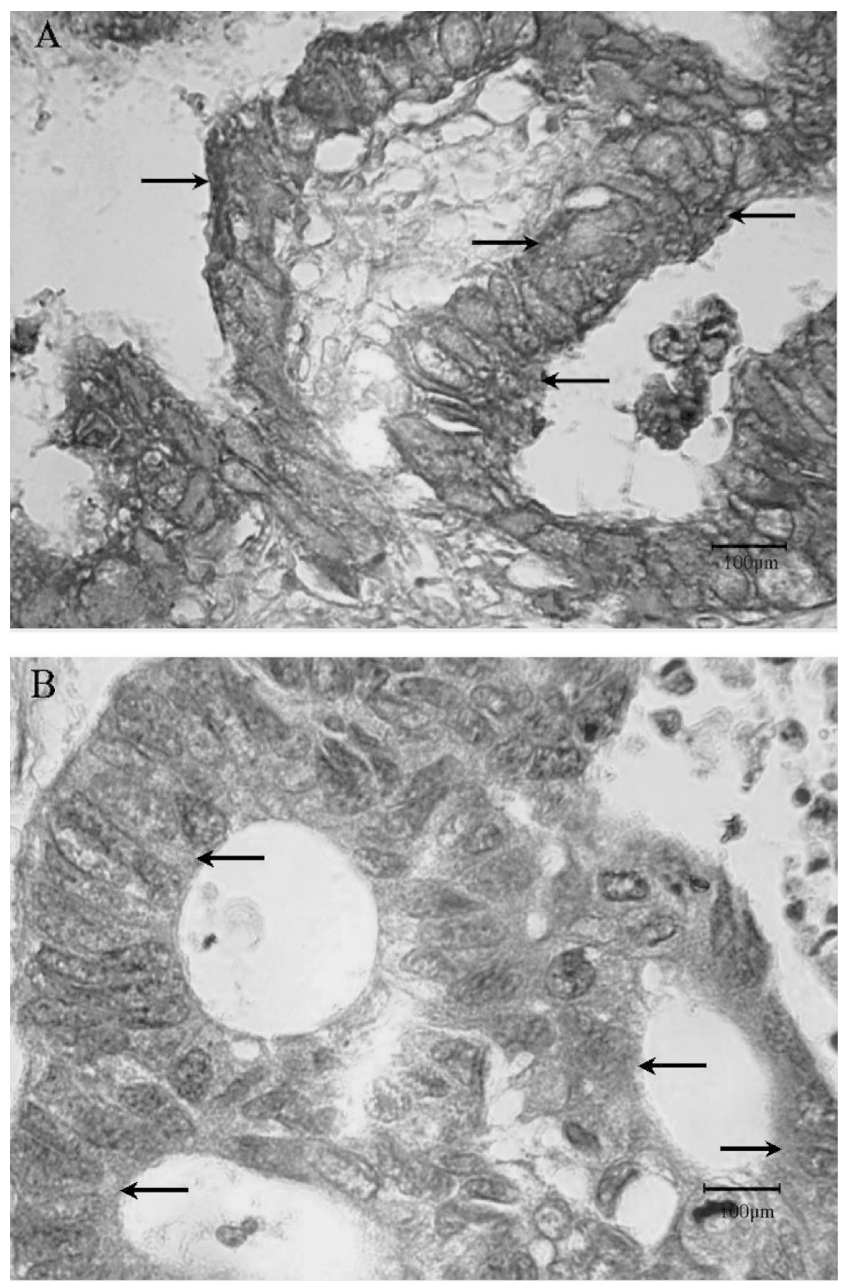

Fig. 1. Immunohistochemistry for HSP72 and gp96 in gastric adenocarcinoma cells (counterstained with hematoxylin), $\times$ 400. Distinctive intra-cytoplasm immunoreactivity was detected for HSP72 (A), and gp96 (B) in gastric cancer cells (arrows).

HSP72, heat shock protein 72; gp96, glycoprotein 96. 
TABLE 1. Relationship between clinicopathology and immunoreactivity of HSP72 and gp96 in gastric carcinomas.

\begin{tabular}{|c|c|c|c|c|c|}
\hline \multirow[t]{2}{*}{ Pathologic types } & \multirow[b]{2}{*}{$n$} & \multicolumn{2}{|c|}{ HSP72 } & \multicolumn{2}{|c|}{ gp96 } \\
\hline & & $-(\%)$ & $+(\%)$ & $-(\%)$ & $+(\%)$ \\
\hline Tissues adjacent to cancers & 60 & $38(63.3)$ & $22(36.7)$ & $45(75.0)$ & $15(25.0)$ \\
\hline Gastric carcinomas $^{\mathrm{a}}$ & 60 & $6(10.0)$ & $54(90.0)$ & $11(18.3)$ & $49(81.7)$ \\
\hline \multicolumn{6}{|l|}{ Tumor Differentiation ${ }^{\mathrm{b}}$} \\
\hline high & 27 & $4(14.8)$ & $23(85.2)$ & $6(22.2)$ & $21(77.8)$ \\
\hline moderate & 20 & $2(10.0)$ & $18(90.0)$ & $3(15.0)$ & $17(85.0)$ \\
\hline low & 13 & $0(0)$ & $13(100)$ & $2(15.4)$ & $11(84.6)$ \\
\hline \multicolumn{6}{|l|}{ Lymph node metastasis } \\
\hline $\mathrm{yes}^{\mathrm{c}}$ & 45 & $0(0)$ & $45(100)$ & $0(0)$ & $45(100)$ \\
\hline no & 15 & $6(40.0)$ & $9(60.0)$ & $11(73.3)$ & $4(26.7)$ \\
\hline \multicolumn{6}{|l|}{ Remote metastasis } \\
\hline yes $^{\mathrm{d}}$ & 34 & $0(0)$ & $34(100)$ & $0(0)$ & $34(100)$ \\
\hline no & 26 & $6(23.1)$ & $20(76.9)$ & $11(42.3)$ & $15(57.7)$ \\
\hline
\end{tabular}

${ }^{\mathrm{a}} p<0.01,{ }^{\mathrm{b}} p<0.01$, vs tissues adjacent to cancers; ${ }^{\mathrm{c}} p<0.05,{ }^{\mathrm{d}} p<0.05$, vs non-metastasis groups.

groups and non-metastasis groups $(p<0.05)$. These results suggest that there exists a significant correlation between expression of both HSP72 and gp96 and progression of gastric carcinomas.

\section{DisCUSSION}

In this study we examined the expression profiles of HSP72 and gp96 in 60 gastric carcinoma samples by immunohistochemistry. The results showed that almost all of the detected gastric carcinomas expressed HSP72, and majority of tumors expressed gp96, which had significant differences compared with that in mucous membrane adjacent to cancers. By means of immunohistochemistry, we found that there was a definite correlation between expression of both HSP72 and gp96 and development of gastric carcinomas.

The HSP family is group of highly conserved proteins synthesized after heat induction or other stressors (Schlesinger 1990; Morimoto 1993; Argon 1999). In mammalian cells, this system is divided into two predominant categories, which appear to be structurally and functionally related: the HSPs and the glucose-regulated proteins (grps) (Schlesinger 1990). During the growth and development of normal cells, HSP70 is constitu- tively expressed at low levels but the expression was dramatically enhanced by stressful conditions (Morimoto 1993). HSP72, belonging to the family of HSP70, is a highly conserved protein synthesized under various stresses. In non-transformed cells at normal conditions, Hsp72 is expressed at very low levels. It is, however, present at elevated levels in the major fraction of tumors and in many transformed cell lines (Kato et al. 2000; Lopez-Cotarelo et al. 2000; Maehara et al. 2000). It is commonly assumed that in tumor cells the expression of HSP72 at elevated levels is the consequence of oncogenic transformation and enhanced expression of HSP72 has a close relationship with epithelial carcinoma cells growth (Volloch and Shelman 1999; Kato et al. 2000; Lopez-Cotarelo et al. 2000). Up-regulated expression of the HSP70 family in tumor cells may be a requirement to serve as molecular chaperones in regulating and stabilizing oncofetal protein and mutant oncogene products during tumor growth process (Maehara et al. 2000; Cardoso et al. 2001; Hwang et al. 2003). In normal cells, gp96 expression could also be induced by various stresses to function as molecular chaperones (Lee 2001; Linderoth et al. 2001). Some researchers 
have implied that enhanced expression of gp96 has a close relationship with cancer cells growth (Wang et al. 2002; Wang et al. 2005a). High level expression of gp96 could contribute to tumorigenicity of certain tumors (Wang et al. 2005a; Fu and Lee 2006). However, few reports have studied the expression of gp96 in gastric carcinomas, especially during the course of tumor growth and differentiation, in simultaneous comparison with HSP72. In these experiments, we found that HSP72 and gp96 were highly expressed when gastric carcinomas progressed, but their roles in gastric carcinoma are not clear. It is reasonable to propose that HSP72 and gp96 up-regulation in these tumor cells are closely related with tumor cell survival and proliferation. Recent studies have suggested that HSPs take part in cell growth and proliferation in several ways such as signal transduction and cell cycle regulation through combining certain proto-oncogene products. This indicates that these proliferating cells need higher level of HSPs to maintain the stability of tumor proteome (Liu et al. 1999; Fisher et al. 2000). It is believed that tumor cells are a group of highly proliferative heterogeneous cells which progress gradually through mutant oncogene products (Renan 1990). Continuous expression of HSP in tumor cells may be required to serve as molecular chaperones in regulating and stabilizing these products during tumor growth process. At the same time, the existence of mutant or oncogene products may stimulate HSP synthesis (Villaseca et al. 1997; Dorsey and Tchounwou. 2003). It has been verified that HSP70 interacts with mutant p53 to stabilize its function; conversely, wild-type p53 may down-regulate HSP70 expression (King et al. 2001). A previous study (Maehara et al. 2000) showed that in differentiated tissue type of gastric cancer, immunohistochemically stained positive cells of HSP70 varied from 0 to $100 \%$. HSP70 expression was related to the differentiated tissue type of gastric cancer, but not to other clinicopathological factors. Our results also showed that the expression of both HSP72 and gp96 in gastric carcinoma was higher than that in tissues adjacent to cancer, but the expression of both HSP72 and gp96 in gastric carcinomas with metastasis was definitely higher than that of gastric carcinomas without metastasis. It is presumed that the combined measurement of HSP72 and gp96 in gastric adenocarcinoma might be more sensitive than that of HSP70 family alone. The results indicate that up-regulation of HSP72 and gp96 is likely to have some relationship with progression, invasion and metastasis of gastric carcinomas.

Studies revealed that considerable expression of HSPs was found in tumor cells, showing that HSPs may be induced by other stresses and participate in broader array of defenses during cell growth and cell differentiation of tumors (Villaseca et al. 1997; Dorsey and Tchounwou 2003; Lebret et al. 2003; Bausero et al. 2004). Thus, it may be presumed that under various stimuli and stressful conditions, in order to avoid the damage caused by deleterious factors such as methylcholanthrene-oncogenesis evocator, gastric mucous membrane has to transcribe and translate high levels of HSPs in order to sustain normal metabolism and functions of cells. Under these conditions, gastric mucous membrane should synthesize HSPs rapidly to exert a protective role for gastric mucous cells. The progression of gastric carcinoma is a gradual process under the longterm influence of various stimuli. During the process, inducible HSP synthesis increases gradually (Isomoto et al. 2003). This viewpoint was confirmed by our results, in that HSP72 and gp96 were expressed at a higher level in gastric carcinoma than that in gastric tissues adjacent to cancer. The expression levels of HSP72 and gp96 may prove useful as diagnostic or prognostic markers for gastric carcinoma.

Numerous investigations have been verified that HSP70 and gp96 are potent stimulators of immune responses (Graner et al. 2003; Castelli et al. 2004; Faure et al. 2004). The classical mechanisms of HSP70 and gp96 against tumors are believed that they may act as chaperones to facilitate major histocompatibility complex-1 (MHC-I) peptide loading, therefore increasing the tumor peptides presented by MHC-I (Wells et al. 1998; Sastry and Linderoth 1999; Berwin and Nicchitta 2001). Studies have shown that HSP72- 
associated peptides can also anchor antigen on the cell membrane and directly present it to natural killer cells or $\gamma \delta \mathrm{T}$ cells as superantigen without being dependent on the stimulation of MHC-I molecules (Multhoff et al. 1997; Zhang et al. 2005). Through this way cytotoxic T lymphocyte responses could be induced and the anti-tumor immunity was activated. Our data show highlevel expression of HSP72 and gp96 in gastric carcinomas, and there was a significant correlation between their expression and progression, metastasis of tumors. These results raise the possibility that expression of HSP72 and gp96 in gastric carcinomas may provide a useful link between immunity and tumor therapy against these cancers.

In conclusion, there is a close correlation between the overexpression of HSP72 and gp96 and progression of gastric carcinomas. The highlevel expression of HSP72 and gp96 may be useful as diagnostic or prognostic markers for gastric carcinoma.

\section{References}

Argon, Y. \& Simen, B.B. (1999) GRP96, an ER chaperone with protein and peptide binding properties. Semin. Cell Dev. Biol., 10, 495-505.

Bausero, M.A., Page, D.T., Osinaga, E. \& Asea, A. (2004) Surface expression of Hsp25 and Hsp72 differentially regulates tumor growth and metastasis. Tumour Biol., 25, 243-251.

Berwin, B. \& Nicchitta, C.V. (2001) To find the road traveled to tumor immunity: the trafficking itineraries of molecular chaperones in antigen-presenting cells. Traffic, 2, 690-697.

Cardoso, F., Di-Leo, A., Larsimont, D., Gancberg, D., Rouas, G., Dolci, S., Ferreira, F., Paesmans, M. \& Piccart, M. (2001) Evaluation of HER2, p53, bcl-2, topoisomerase II-alpha, heat shock proteins 27 and 70 in primary breast cancer and metastatic ipsilateral axillary lymph nodes. Ann. Oncol., 12, 615-620.

Castelli, C., Rivoltini, L., Rini, F., Belli, F., Testori, A., Maio, M., Mazzaferro, V., Coppa, J., Srivastava, P.K. \& Parmiani, G. (2004) Heat shock proteins: biological functions and clinical application as personalized vaccines for human cancer. Cancer Immunol. Immunother., 53, 227-233.

Dorsey, W.C. \& Tchounwou, P.B. (2003) CYP1a1, HSP70, $\mathrm{P} 53$, and c-fos expression in human liver carcinoma cells (HepG2) exposed to pentachlorophenol. Biomed. Sci. Instrum., 39, 389-396.

Faure, O., Graff-Dubois, S., Bretaudeau, L., Derre, L., Gross, DA., Alves, PM., Cornet, S., Duffour, M.T., Chouaib, S., Miconnet, I., Gregoire, M., Jotereau, F., Lemonnier, F.A., Abastado, J.P. \& Kosmatopoulos, K. (2004) Inducible Hsp70 as target of anticancer immunotherapy: Identification of HLA-A*0201-restricted epitopes. Int. J. Cancer,
108, 863-870.

Fisher, D.L., Mandart, E. \& Doree, M. (2000) Hsp90 is required for c-Mos activation and biphasic MAP kinase activation in Xenopus oocytes. EMBO J., 19, 1516-1524.

Fu, Y. \& Lee, A.S. (2006) Glucose regulated proteins in cancer progression, drug resistance and immunotherapy. Cancer Biol. Ther., 5, 741-744.

Gabai, V.L., Budagova, K.R. \& Sherman, M.Y. (2005) Increased expression of the major heat shock protein Hsp72 in human prostate carcinoma cells is dispensable for their viability but confers resistance to a variety of anticancer agents. Oncogene, 24, 3328-3338.

Garcia-Bermejo, L., Vilaboa, N.E., Perez, C., Galan, A., De-BlaS, E. \& Aller, P. (1997) Modulation of heat-shock protein 70 (HSP70) gene expression by sodium butyrate in U-937 promonocytic cells: relationships with differentiation and apoptosis. Exp. Cell Res., 236, 268-274.

Graner, M.W., Zeng, Y., Feng, H. \& Katsanis, E. (2003) Tumorderived chaperone-rich cell lysates are effective therapeutic vaccines against a variety of cancers. Cancer Immunol. Immunother., 52, 226-234.

Hwang, T.S., Han, H.S., Choi, H.K., Lee, Y.J., Kim, Y.J., Han, M.Y. \& Park, Y.M. (2003) Differential, stage-dependent expression of Hsp70, Hsp110 and Bcl-2 in colorectal cancer. J. Gastroenterol. Hepatol., 18, 690-700.

Isomoto, H., Oka, M., Yano, Y., Kanazawa, Y., Soda, H., Terada, R., Yasutake, T., Nakayama, T., Shikuwa, S., Takeshima, F., Udono, H., Murata, I., Ohtsuka, K. \& Kohno, S. (2003) Expression of heat shock protein (Hsp) 70 and Hsp 40 in colonic cancer. Cancer Lett., 198, 219-228.

Kato, K., Yamanaka, K., Nakano, M., Hasegawa, A. \& Okada, S. (2000) 72-kDa stress protein (hsp72) induced by administration of dimethylarsinic acid to mice accumulates in alveolar flat cells of lung, a target organ for arsenic carcinogenesis. Biol. Pharm. Bull., 23, 1212-1215.

King, F.W., Wawrzynow, A., Hohfeld, J. \& Zylicz, M. (2001) Co-chaperones Bag-1, Hop and Hsp40 regulate Hsc70 and Hsp90 interactions with wild-type or mutant p53. EMBO J., 20, 6297-6305

Lebret, T., Watson, R.W., Molinie, V., O’neill, A., Gabriel, C., Fitzpatrick, J.M. \& Botto, H. (2003) Heat shock proteins HSP27, HSP60, HSP70, and HSP90: expression in bladder carcinoma. Cancer, 98, 970-977.

Lee, A.S. (2001) The glucose-regulated proteins: stress induction and clinical applications. Trends Biochem. Sci., 26, 504-510.

Linderoth, N.A., Simon, M.N., Rodionova, N.A., Cadene, M., Laws, W.R., Chait, B.T. \& Sastry, S. (2001) Biophysical analysis of the endoplasmic reticulum-resident chaperone/ heat shock protein gp96/GRP96 and its complex with peptide antigen. Biochemistry, 40, 1483-1495.

Liu, H., Vuyyuru, V.B., Pham, C.D., Yang, Y. \& Singh, B. (1999) Evidence of an interaction between Mos and Hsp70: a role of the Mos residue serine 3 in mediating Hsp70 association. Oncogene, 18, 3461-3470.

Lopez-Cotarelo, C., Sellhaus, B., Baba, H.A., Manegold, E., Luka, J., Handt, S., Mittermayer, C., Klosterhalfen, B. \& Tietze, L. (2000) Expression of heat shock proteins 72/73 in human peritoneal mesothelial cells in vivo and in vitro. Nephron., 85, 148-155.

Maehara, Y., Oki, E., Abe, T., Tokunaga, E., Shibahara, K., Kakeji, Y. \& Sugimachi, K. (2000) Overexpression of the heat shock protein HSP70 family and p53 protein and prognosis for patients with gastric cancer. Oncology, 58, 
144-151.

Morimoto, R.I. (1993) Cells in stress: transcriptional activation of heat shock genes. Science, 259, 1409-1410.

Multhoff, G., Botzler, C., Jennen, L., Schmidt, J., Ellwart, J. \& Issels, R. (1997) Heat shock protein 72 on tumor cells: a recognition structure for natural killer cells. J. Immunol., 158, 4341-4350.

Renan, M.J. (1990) Cancer genes: current status, future prospects, and applications in radiotherapy/oncology. Radiother. Oncol., 19, 197-218.

Sastry, S. \& Linderoth, N. (1999) Molecular mechanisms of peptide loading by the tumor ejection antigen/heat shock chaperone gp96 (GRP96). J. Biol. Chem., 274, 12023-12035.

Schlesinger, M.J. (1990) Heat shock proteins. J. Biol. Chem., 265, 12111-12114.

Villaseca, M.A., Roa, I., Araya, J.C., Roa, J.C. \& Flores, P. (1997) Double immunostaining for p53 and molecular chaperone hsp72/73 in gastric carcinoma. Mol. Pathol., 50, 317-321.

Volloch, V.Z. \& Sherman, M.Y. (1999) Oncogenic potential of Hsp72. Oncogene, 18, 3648-3651.
Wang, Q., An, L., Chen, Y. \& Yue, S. (2002) Expression of endoplasmic reticulum molecular chaperon GRP96 in human lung cancer tissues and its clinical significance. Chin. Med. J., 115, 1615-1619.

Wang, Q., He, Z., Zhang, J., Wang, Y., Wang, T., Tong, S., Wang, L., Wang, S. \& Chen, Y. (2005a) Overexpression of endoplasmic reticulum molecular chaperone GRP94 and GRP78 in human lung cancer tissues and its significance. Cancer Detect. Prev., 29, 544-551.

Wang, X.P., Liu, G.Z., Song, A.L., Chen, R.F., Li, H.Y. \& Liu, Y (2005b) Expression and significance of heat shock protein 70 and glucose-regulated protein 94 in human esophageal carcinoma. World J. Gastroenterol., 11, 429-432.

Wells, A.D., Rai, S.K., Salvato, M.S., Band, H. \& Malkovsky, M. (1998) Hsp72-mediated augmentation of MHC class I surface expression and endogenous antigen presentation. Int. Immunol., 10, 609-617.

Zhang, H., Hu, H., Jiang, X., He, H., Cui, L. \& He, W. (2005) Membrane HSP70: the molecule triggering gammadelta T cells in the early stage of tumorigenesis. Immunol. Invest., 34, 453-468. 